\title{
Die FMH kennen, um sie nutzen zu können
}

\author{
Anne-Geneviève Bütikofera, Jacqueline Wettstein ${ }^{b}$ \\ ${ }^{a}$ Generalsekretärin der $\mathrm{FMH} ;{ }^{\mathrm{b}}$ Leiterin der Abteilung Kommunikation der FMH
}

Was die FMH konkret macht, ist eine immer wieder gestellte Frage. Aus diesem Grund lud der nationale Berufsverband Ärztinnen und Ärzte, welche sich neu in der Standespolitik engagieren, und Geschäftsstellenleiter von Ärzteorganisationen zu einer halbtägigen Veranstaltung ein. Diese bot Gelegenheit, die Aktivitäten der FMH kennenzulernen und sich zu aktuellen gesundheitspolitischen Themen auszutauschen.

Wer zum Beispiel das Präsidium einer Fachgesellschaft übernimmt, Vorstandsmitglied einer Dachorganisation wird oder die Geschäftsstelle einer Kantonalgesellschaft leitet, darf sich auf spannende, vielseitige Aufgaben freuen. Um neu gewählten und künftigen Amtsträgerinnen und -trägern die FMH in ihrer Funktionsweise und ihren Aktivitäten vorzustellen, lud sie die FMH-Generalsekretärin zum zweiten Mal zu einer halbtägigen Einführungsveranstaltung ein. Kurzreferate sowie Workshops boten Gelegenheit, sich zu aktuellen standespolitischen Themen zu informieren und auszutauschen.

\section{Für die Freiheit und Unabhängigkeit des Arztberufes}

Mit über 70 Ärzteorganisationen und rund 40000 Mitgliedern ist die FMH heute einer der grossen berufspolitischen Verbände und gehört im Schweizer Gesundheitswesen zu den gewichtigen Akteuren. Der erste Schweizer Ärzteverband entstand bereits zur Zeit der

\section{Der erste Schweizer Ärzteverband entstand} bereits zur Zeit der Französischen Revolution.

Französischen Revolution; im 18. Jahrhundert bildeten sich verschiedene regionale Ärzteverbände, die sich mit der Zeit zu interkantonalen Ärzteorganisationen zusammenschlossen. 1901 wurde schliesslich der Grundstein für die FMH gelegt, ab 1930 entstanden die verschiedenen medizinischen Fachgesellschaften. Allen diesen Organisationen war und ist etwas gemeinsam: Ärztinnen und Ärzte sollen ihren Beruf frei, unabhängig und eigenverantwortlich ausüben können.
Die FMH vertritt als Dachorganisation der in der Schweiz tätigen Ärzteschaft die Interessen ihrer Mitglieder gegenüber Behörden und weiteren Institutionen sowie auch gegenüber der Bevölkerung. Für eine

In zahlreichen Workshops lernten die Teilnehmenden die verschiedenen Dienstleistungen der FMH kennen.

ausgewogene Vertretung aller Mitgliederinteressen sorgen verschiedene Organe innerhalb der FMH: die Ärztekammer, welche als Parlament die Grundzüge der Verbandspolitik bestimmt; die Delegiertenversammlung, die alle wichtigen gesundheits- und standespolitischen Fragen behandelt; der Zentralvorstand, der die FMH gegenüber Öffentlichkeit und Behörden vertritt; sowie die Urabstimmung, die als wichtigstes Organ von allen stimmberechtigten Mitgliedern analog einer Volksabstimmung direktdemokratisch genutzt werden kann.

\section{Gouverner, c'est prévoir}

Gemäss dem vom FMH-Präsidenten zitierten Leitspruch «Gouverner, c'est prévoir» hat die FMH vorausschauend die Standesordnung geschaffen. Als Verhaltenskodex für ihre Mitglieder definiert sie die wichtigsten Berufspflichten und verschiedene berufsethische Regeln, wie etwa den Umgang mit Sterbenden oder die Zusammenarbeit von Ärzteschaft und Industrie. Damit stellt die Standesordnung eine Art Qualitätslabel dar. Umsichtig hat die FMH agiert, als aufgrund der bilateralen Verträge vor zehn Jahren auf der Kippe stand, ob sie die Weiter- und Fortbildung von 
Ärztinnen und Ärzten weiterhin zentral mitgestalten kann. Zu diesem Zweck schuf der Berufsverband 2009 das Schweizerische Institut für ärztliche Weiter- und Fortbildung SIWF. Als selbständiges Organ der FMH betreut das SIWF jährlich über 10000 Assistenzärztinnen und -ärzte während ihrer Weiterbildung und zertifiziert rund 1500 Weiterbildungsstätten. Das SIWF hat sich und seine Tätigkeiten anlässlich der Einführungsveranstaltung ebenfalls vorgestellt.

\section{Zahlreiche Projekte, nützliche Dienst- leistungen}

In zahlreichen Workshops lernten die Teilnehmenden die verschiedenen Dienstleistungen der FMH kennen und informierten sich über aktuelle Arbeiten der FMH. Das Projekt TARCO (TARMED Consensus) beispielsweise will mit allen in der Ärztekammer vertretenen Organisationen einen gemeinsamen Nenner finden, um die ambulante Tarifrevision fortzuführen. Neben der laufenden Weiterentwicklung von SwissDRG engagiert sich die FMH auch bei der Ausgestaltung weiterer stationärer Tarife wie ST Reha, dem Tarifsystem für die stationäre Rehabilitation, und TARPSY, dem Tarifsystem für die stationäre Psychiatrie. Dank Lobbying hat die FMH insbesondere erreicht, dass bei TARPSY auf Fallpauschalen verzichtet und stattdessen Tagespauschalen gewählt wurden. Ebenso erfuhren die Teilnehmenden, wie sich die FMH auf nationaler Ebene zu Public Health einbringt, welche Ziele die Qualitäts-Charta der SAQM verfolgt und wie die FMH mit der Interprofessionellen Arbeitsgruppe IPAG EPD das elektronische Patientendossier zum Nutzen von Ärztinnen und Patienten mitgestaltet. Ver- schiedene Informationsstände stellten Angebote wie den Rechtsleitfaden, die gesundheitspolitischen Empfehlungen der FMH oder das Online-Ärzteverzeichnis doctorfmh.ch vor und orientierten eingehend über die Standeskommission, über die Gutachterstelle der FMH sowie über das MPA-Berufsbild.

\section{Schlussplenum zu aktuellen Brennpunkten}

Den Abschluss der Veranstaltung bildete das gemeinsame Schlussplenum, dessen Inhalte die Teilnehmenden selbst bestimmten. Sie nahmen die Gelegenheit wahr, sich sowohl über den Stand der ambulanten Tarifrevision als auch über die Umsetzung des Bundesgesetzes über das elektronische Patientendossier zu orientieren. Besonders kritisch äusserten sich die Teil-

\section{Den Abschluss der Veranstaltung bildete} das gemeinsame Schlussplenum, dessen Inhalte die Teilnehmenden selbst bestimmten.

nehmenden gegenüber dem Projekt MARS des Bundes, welches gemäss gesetzlicher Vorgabe die Arztpraxen und ambulanten Zentren zur Datenlieferung verpflichtet. Die FMH wird sich wie bis anhin dafür einsetzen, ihre Mitglieder bestmöglich zu unterstützen und den Aufwand zu minimieren. Betreffend Vernehmlassung zum Datenschutzreglement konnte sich die FMH bereits erfolgreich durchsetzen und erreichte eine deutliche Fristverlängerung.

Wir freuen uns, Sie zahlreich an der nächsten Einführungsveranstaltung begrüssen zu dürfen. 